\title{
Simple multiwavelength Brillouin-Erbium-doped fiber laser structure based on short SSMF
}

\begin{abstract}
An efficient multiwavelength Brillouin-Erbium fiber laser (BEFL) was developed by improving the feedback mechanism of Brillouin Stokes lines. The BEFL performance in terms of threshold power and wavelengths count was improved by using a new structure of double pass amplification cavity. The new structure utilized the variable optical coupler (VOA) not only as the input and output ports but also to form a fiber loop mirror that reduces the number of optical components, thus, only three optical components were needed. By optimizing the Brillouin and $1480 \mathrm{~nm}$ pump power, up to 41 channels and 26 channels were obtained using $0.6 \mathrm{~km}$ and $0.3 \mathrm{~km}$ long of standard single mode fiber (SSMF), respectively.
\end{abstract}

Keyword: Fiber lasers; Fiber; Stimulated Brillouin scattering; Rayleigh scattering; Nonlinear optics 